\begin{tabular}{|c|c|}
\hline Title & $\begin{array}{l}\text { Context-Aware and DRM-Enabled Content Adaptation Platform for Collaboration } \\
\text { Applications }\end{array}$ \\
\hline Authors & $\begin{array}{l}\text { Anna Carreras }{ }^{1}, \text { Vitor Barbosa }^{2}, \text { Hemantha Kodikara Arachchi }^{3}, \text { Safak Dogan }^{3} \text {, } \\
\text { Maria Teresa Andrade }^{2,4}, \text { Jaime Delgado }^{1}, \text { Eva Rodríguez }^{1}, \text { Ahmet M. Kondoz }^{3}\end{array}$ \\
\hline $\begin{array}{l}\text { Affiliations } \\
\text { and } \\
\text { contacts }\end{array}$ & $\begin{array}{l}{ }^{1} \text { Universitat Politecnica de Catalunya, Departament d'Arquitectura de } \\
\text { Computadors, Campus Nord, Modul D6, Jordi Girona 1-3, E-08034 Barcelona, } \\
\text { Spain } \\
\text { \{annac, jaime.delgado, evar\}@ac.upc.edu } \\
{ }^{2} \text { INESC Porto, Rua Dr. Roberto Frias 378, 4200-465 Porto, Portugal } \\
\text { \{vhsb, maria.andrade\}@inescporto.pt } \\
{ }^{3} \text { I-Lab, Centre for Communication Systems Research (CCSR), University of } \\
\text { Surrey, Guildford, GU2 7XH, Surrey, UK } \\
\{\text { H.Kodikaraarachchi, S.Dogan, A.Kondoz\}@surrey.ac.uk } \\
{ }^{4} \text { Faculty of Engineering of the University of Porto (FEUP), Rua Dr. Roberto Frias } \\
\text { s/n, 4200-465 Porto, Portugal } \\
\text { mandrade@fe.up.pt }\end{array}$ \\
\hline
\end{tabular}




\title{
Context-Aware and DRM-Enabled Content Adaptation Platform for Collaboration Applications
}

\begin{abstract}
This paper presents a context-aware and Digital Rights Management (DRM)enabled content adaptation platform developed within VISNET II Network of Excellence. Based on MPEG-21 Digital Item Adaptation (DIA) and a profiling approach, a flexible technique is presented to express context. A new type of context, integrating DRM and content adaptation, is also identified. An ontology model is proposed to take adequate adaptation decisions to satisfy user expectations. The use of standards and ontologies, and the distributed modular architecture of the platform guarantee interoperability, flexibility and scalability. The applicability of this platform is presented in the context of a Virtual Classroom scenario.
\end{abstract}

Index Terms - Context-aware content adaptation, ontology-based and DRM-enabled adaptation decision, adaptation authorization, MPEG-21, Web Services-based interfaces, collaboration application

\section{INTRODUCTION}

$\mathrm{P}$ roliferation of numerous multimedia applications in everyday life, such as mobile video, video-on-demand, e-learning, e-health, gaming, etc, have fuelled the access to rich content in diverse usage environments. Nowadays, users wish to access any type of digital content anywhere and anytime. This notion, which has received a great deal of attention from the research community recently, is referred to as Universal Multimedia Access (UMA) [1]. The majority of the research activities reported in this area focus mainly on content adaptation [1], [2]. Nevertheless, the use of contextual information is also equally essential to achieve efficient and useful adaptations that enrich the user experience.

Furthermore, the explosion in the number of people creating multimedia items and the desire to protect their created contents against unauthorized use pose a significant demand for some means of governance to context-based adaptations.

Bearing the aforementioned issues in mind, this paper presents a proposed scalable and modular platform for context-aware and Digital Rights Management (DRM)-enabled adaptation of multimedia content. The sequence of events that take place and the corresponding messages required while performing an adaptation operation are also described. The innovative character of this platform is demonstrated by adopting a number of new approaches, such as: 
- Combining the use of ontologies and low-level context to drive the adaptation decision taking process.

- Verifying and enforcing usage rights within the adaptation operations.

- Incorporating multi-faceted adaptation tools, so as to provide a wide range of on-the-fly and on-demand adaptation operations that suit various dynamic requirements.

The platform is also featured with a new type of contextual information [3] derived from integrating DRM with context-based content adaptation. In turn, all of the aforementioned features supported by this adaptation platform enable addressing various aspects of the seamless delivery of networked multimedia contents in the wider sense.

This paper is organized as follows. Following an overview of the state-of-the-art in Section II, Section III discusses the types and representation of context, presenting an innovative approach of using contextual information to represent adaptation authorization. The use of ontologies for enriching context-based adaptation is elaborated in Section IV. Section V describes the architecture of the context-aware and DRM-enabled content adaptation platform developed within the VISNET II Network of Excellence (NoE) project [4]. Section VI focuses on presenting the necessary interfaces between the several modules of this platform. This section also discusses a Virtual Classroom scenario [5] as a potential use case for the application of the proposed platform, so as to better elaborate the results presented in the paper. Finally, Section VII concludes the paper.

\section{STATE-OF-THE-ART IN CONTEXT-AWARE CONTENT ADAPTATION}

The use of contextual information is instrumental for the successful implementation of useful and meaningful content adaptation operations. These, in turn, are becoming extremely important for the implementation of systems and platforms that deliver seamless multimedia services to the end-user. Content adaptation has in fact already gained considerable importance in today's multimedia communications, and will certainly become an essential functionality of any service, application or system in the near future. The continuing advances in technology will only emphasize the great heterogeneity that exists today in devices, systems, services and applications. Likewise, this will also bring out the desires of consumers for more choices, better quality and more personalization options. But, to empower those systems to perform meaningful content adaptation operations that meet users' expectations and satisfy their usage environment constraints, it is imperative that they use contextual information, and thus take decisions based on that information. Research on context awareness started more than a decade ago [6]. However, it was only recently that this concept gained increased popularity among the multimedia research community, and also started to be addressed at the standardization level.

\section{A. Concepts and models for context}

Early definitions of context were limited or specific to a given application, as they were usually made by examples of the type of context information being used. Research work around context-aware services was initiated especially in the mobile applications area. It was mainly 
focused on processing information regarding the location and the type of device being used to receive and present the content. The work evolved, not only in the mobile communications area, but also in other areas. Other types of information collected through varied types of sensors also started to be used. One example is the area of Human Computer Interfaces (HCI), where information regarding user gender and age, his/her emotions, disabilities or environmental conditions was used to adapt the application's interface to particular usages.

Dey [7] provides a good generic definition of context that can be applied no matter what the type of information being used or the application in view may be. This is probably the most quoted definition of context:

"Context is any information that can be used to characterize the situation of an entity. An entity is a person, place, or object that is considered relevant to the interaction between a user and an application, including the user and application themselves."

This definition implicitly states that any application using additional information with the capacity to condition the way the user interacts with the content is a context-aware application.

Other popular definitions can be found in [8], [9], and [10]. Nevertheless, they are too wide for our purposes. In our opinion, contextual information can be any kind of information that characterizes or provides additional information regarding any feature or condition of the complete delivery and consumption environment.

This diversity of information can be classified in many different ways. Our approach is aligned with our previous definition of context and distinguishes between descriptors associated to each of the entities involved in the delivery and consumption of the multimedia content. Nevertheless, other dimensions of the contextual information could be considered. These other dimensions refer, for example, to the characteristics and nature of the contextual information itself and not to the type of entity that it describes. As an example, [7] and [11] classified context into four categories, named: Activity, Identity, Location, and Time.

Explicit contextual information is generated by sensors. This kind of information is also known as "low-level context". Any hardware appliance, software component or entity capable of generating data describing some aspect of the context of usage can be designated as a sensor. Examples of low-level context are a measurement of the available bandwidth performed by a network probe, dimensions of a terminal display, a temperature value obtained by a thermometer, an indication of available Central Processing Unit (CPU) resources of a computer, an alarm generated by a motion detection sensor, etc. Gathering, representing and using different kinds of contextual information are essential steps of any system aiming to provide adapted services and content which satisfy usage constraints while meeting users' expectations.

\section{B. Standardization efforts on contextual information for content adaptation}

The representation of context is still an open research issue. In order to guarantee interoperability for UMA scenarios, a standardized format should be used. Two important initiatives are the Composite Capability/Preference Profile (CC/PP) [12] and the User Agent Profile (UAProf) [13].

The former, created by the World Wide Web Consortium (W3C) [14], defines a Resource 
Description Framework (RDF) for describing device capabilities and user preferences. It provides the means to specify client capabilities (i.e., the "user agent" information) and user preferences using Uniform Resource Identifiers (URIs) and RDF text sent in Hypertext Transfer Protocol (HTTP) requests. The user agent specifies the preferences of the user in the header of the client HTTP request, such as versions of content or languages, and is empowered with negotiation capabilities. Although the CC/PP specification was originally developed to be used mainly on wireless devices, it can be applied to any Web-enabled terminal. It uses RDF in its eXtensible Markup Language (XML) serialized format to exchange profiles between devices with information on the user agent's capabilities and the user's preferences. CC/PP uses a vocabulary to define the format and language for specifying the names and values of components as well as their attributes. However, different CC/PP profiles or applications may use different vocabularies. This is a particular feature of CC/PP that allows different applications to define and use particular vocabularies that suit their particular needs. One of the vocabularies that has been traditionally used in CC/PP profiles is the UAProf in the mobile world. For other application areas, and in general terms, the $\mathrm{W} 3 \mathrm{C}$ recommends the use of RDF to define vocabularies. In spite of its wide applicability, the CC/PP model presents some limitations when addressing more complex context-aware scenarios. Although in principle any kind of contextual information could be described using CC/PP as long as the context information could be described using $\mathrm{RDF}$, it does not provide the mechanisms to carry additional information, such as temporal information or resolution of the contextual information. However, the most important limitations may come from the fact that CC/PP Components and Attributes (or subtypes of them) have a limited set of values and a restricted syntax. For example, CC/PP does not provide any support for expressing relationships or constraints. It also has some limitations regarding the type of information.

The latter (i.e., UAProf), developed by the Open Mobile Alliance [15], provides an open vocabulary for Wireless Access Protocol (WAP) clients to communicate their capabilities to servers. It defines the data structure to describe client devices and to transport that information to the servers. This information may include hardware characteristics, such as screen size or type of keyboard, software characteristics, such as browser manufacturer, and also user preferences (e.g., sound enabled, color choices etc). The idea is to empower the service providers with information that may assist them in customizing the services to the end-user needs to a certain extent. The UAProf vocabulary has six components:

- HardwarePlatform: characteristics of the hardware of the terminal, including the type of device, model, display size or memory.

- SoftwarePlatform: characteristics of the operating environment of the device.

- NetworkCharacteristics: information about the network infrastructure, such as bearer information.

- BrowserUA: identification of the browser application available on the device.

- WapCharacteristicslists: the WAP capabilities of the terminal.

- PushCharacteristics: push specifications of the device, such as maximum size of a push message. 
However, both initiatives are limited to specific application domains, and represent a small subset of contextual information. To date, the most complete initiative to represent context for generic multimedia applications has been achieved by the MPEG community by means of the $7^{\text {th }}$ part of its MPEG-21 standard. It is called MPEG-21 Digital Item Adaptation (DIA) [16], and includes description tools to facilitate context-based content adaptation. In addition to supporting the description of terminal capabilities and user preferences, as the aforementioned specifications, MPEG-21 DIA addresses the static and dynamic characterization of networks and the description of natural environments, providing the means to express them as context constraints. It also allows relating possible content adaptation operations or sets of service parameters to the expected results in terms of quality. Finally, and of utmost importance to our work, MPEG-21 also specifies DRM-related tools. Among the MPEG-21 DIA specified tools, the Usage Environment Description (UED) tool provides a complete set of context descriptors. Therefore, UED is the main focus of our work and will be discussed in more detail in Section III.

MPEG-21 [17] is the ISO/IEC standard currently under its final phase of development in MPEG. It focuses on the development of an extensive set of specifications, descriptions and tools to facilitate the transactions of multimedia content in heterogeneous network environments. The standard is currently divided into 18 parts. The basic concepts of MPEG-21 are the User and the Digital Item (DI). The User is any type of actor that manipulates content, be it a person or a system (e.g., subscriber, producer, provider, network). A DI is the smallest unit of content for transaction and consumption, and at the conceptual level, it can be seen as a package of multimedia resources related to a certain subject (e.g., MPEG-2 video stream, text file, etc), together with associated descriptions (e.g., rights descriptions, other context descriptors, MPEG7 audio and video descriptors, etc).

\section{Integration of DRM and adaptation}

Although DRM [18], [19] and adaptation [20], [21] have been intensively researched in the recent years, these two areas have always been studied independently.

In governed multimedia networks [22], the rights and permissions related to the use of content can be expressed by means of a Rights Expression Language (REL). However, RELs developed so far are not sufficient considering that adaptation has already gained significant importance in end-to-end multimedia services. Accordingly, new descriptors are required to express permissible conversions, and also to govern content adaptations [3].

The only standardization initiative trying to integrate both research areas together comes from the MPEG community, which includes specifications for DRM and content adaptation within one of its standards, the MPEG-21. On one hand, part 5 of the MPEG-21 standard specifies a rights expression language, the MPEG-21 REL [23], for outlining users' rights to act on a digital content. On the other hand, part 7: MPEG-21 DIA [16], provides support for content adaptation, which together with MPEG-21 REL can be used to define rights expressions to govern adaptation in an interoperable way.

The data model defined for MPEG-21 REL licenses includes four basic entities:

- The principal to whom the grant is issued. 
- The right that the user can exercise.

- The resource to which the right in the grant applies.

- The conditions that must be met before the right can be exercised.

The basic relationship among these entities is specified by means of the MPEG-21 REL assertion "grant". A full rights expression is called a license. It includes a grant or a set of grants, and an issuer element that contains issuer specific details and the digital signature for the license (see Table 1 for an example). To guarantee interoperability, MPEG-21 DIA conversion permissions have to be integrated within the Condition field of each grant. The details of this integration will be discussed in Section III.B.

Although real implementations of adaptation authorization have not been deployed yet, projects currently working with MPEG-21 DIA, such as DAIDALOS [24] and aceMEDIA [25], have earmarked this aspect as possible future work. Other projects, such as AXMEDIS [26], the second part of Projecte Integrat, named project Machine [27], and VISNET II NoE [4] are already actively exploring this area.

\section{Ontologies in context-aware content adaptation}

Until recently, one of the main challenges faced by context-aware applications has been the lack of standardized models to gather, represent, and process contextual information. Another difficulty consisted in incorporating knowledge to automate the process of combining contextual information from different sources and extracting conclusions to trigger reactive measures, as the same information may lead to different conclusions. Thus, the system that is analyzing the sensed context should provide a common contextual information representation, which allows to check for information consistency and to derive complex contextual information [28]. Consequently, the new generation of context-aware systems is looking at the aspect of reasoning about the sensed context. This is being done through the use of models with explicit rules and relations to allow combining explicit context according to the application under consideration [29], [30]. In advanced context-aware systems, the use of context involves three basic sets of activities: sensing the low-level context, building higher-level context and sensing changes in the context [31]. Low-level context is generated by sensors as indicated in Section II.A. Building higher-level context refers to the ability to establish relationships among sensed or low-level context to build understanding at higher levels of conceptual abstractions, the way humans do. For example, sensors such as a network probe, a microphone and a Global Positioning System (GPS) receiver may generate values of the instantaneous available bandwidth, of the sound amplitude and of geographical coordinates. All of these values correspond to low-level or sensed context. Using this low-level information, it may be possible to infer the abstract concept, such as "a crowded train station". Sensing context changes should be a continuous process, enabling systems to react to variations of the context of usage during the service lifetime.

The adoption of an ontology-based approach to model context provides a powerful means to describe different contexts in a semantically richer form, thus allowing a finer and more accurate characterization of the situation the user is in while consuming multimedia content. Although there is not a universal consensus on the definition of ontology, it is generally accepted that 
ontology is a specification of a conceptualization. In practice, an ontology allows the formal representation of concepts (classes of subjects) and their interrelationships. Concepts are the entities on the abovementioned Dey's definition of context. The ontology thus provides the means to formally represent different types of contextual information and to establish specific rules on how to relate that information. This formal framework allows the analysis of the domain of knowledge (the context of usage) through reasoning in a machine-interpretable way, providing the means for machines to use low-level information to derive high-level concepts in an automatic way.

\section{TYPES AND REPRESENTATION OF CONTEXT}

Our work performed in VISNET II NoE concerning context-aware content adaptation systems is based on the assumption that a standardized representation of the contextual information is used. This aspect is considered as instrumental to enable interoperability among systems and applications, and across services. As introduced in Section II.B, MPEG-21 DIA seems to be the most complete standard, and thus the ideal candidate for exploiting in this work. MPEG-21 DIA defines the UED tool, which is a full set of contextual information that can be applied to any type of multimedia system, as it assures device-independence. UED includes the description of terminal capabilities and network characteristics as well as User characteristics and preferences, and natural environment conditions. Furthermore, the MPEG-21 DIA standard specifies appropriate XML schemas to represent the contextual information.

\section{A. Profiling}

Contextual profiles have the potential to simplify the generation and use of context by creating restricted groups of contextual descriptions from the full set of DIA UEDs. Each group or profile contains only the descriptions that are essential to a specific application scenario. This approach also promotes interoperability, as different context providers are able to generate the same type of contextual information as their counterparts that use the same standard representation.

UED is divided into four main blocks, each of which is associated to an entity or concept within the multimedia content chain: User, Terminal, Network and Natural Environment. We believe that this division is a good starting point to define profiles. The identification of profiles inside each class can potentially simplify the use of these standardized descriptions by different entities involved in the provision of context-aware multimedia services, and thus can increase its rate of acceptance. Accordingly, our initial proposal for the definition of profiles is also based on the four existing classes: User profile, Terminal profile, Network profile, and Natural Environment profile. Each of these profiles consists of sets of the corresponding elements of MPEG-21 DIA UED to assure a full compliance with the standard. A detailed description of these profiles can be found in [5].

Together with the aforementioned contextual information, the description of the multimedia resources is also required to drive the adaptation decision taking process. The content descriptions are expressed in terms of MPEG-7 Multimedia Description Schemes (MDSs) [32] metadata, including information about media encoding, media bit rate, spatial resolution, frame 
rate, etc.

B. Adaptation authorization: a new type of context

In governed networks, adaptation operations should only be performed if they do not violate any condition expressed in the licenses. The first Amendment of MPEG-21 DIA, named Conversions and Permissions, can be divided into two main parts. The first part specifies the description formats for multimedia conversion capabilities, which offer description tools for the conversions that a terminal is capable of performing. The second part specifies description formats for permissions and conditions for multimedia conversions, which are useful to determine what kind of adaptations are permitted under what kind of conditions. We focus on the second part, and present means of filling the gap between DIA and REL/RDD by embedding adaptation descriptions into rights expressions.

Table I presents the schema of a license that allows defining permissible changes and the associated constraints. It provides the mechanisms to specify which changes are allowed (permittedDiachanges - ConversionDescription) and the conditions under which those changes can be performed (changeconstraint).

The general structure of the license in Table I is based on MPEG-21 REL. As introduced in Section II, we can identify the ContentProducer (as the issuer of the license), the User (as the principal to whom the grant is issued), the play (as the right that can be exercised by the user), the Video (as the resource to which the right in the grant applies), and the conditions that must be met before the right can be exercised. Inside the allConditions field, the aforementioned MPEG21 DIA descriptors can be noticed.

Table I. Schema of a MPEG-21 license

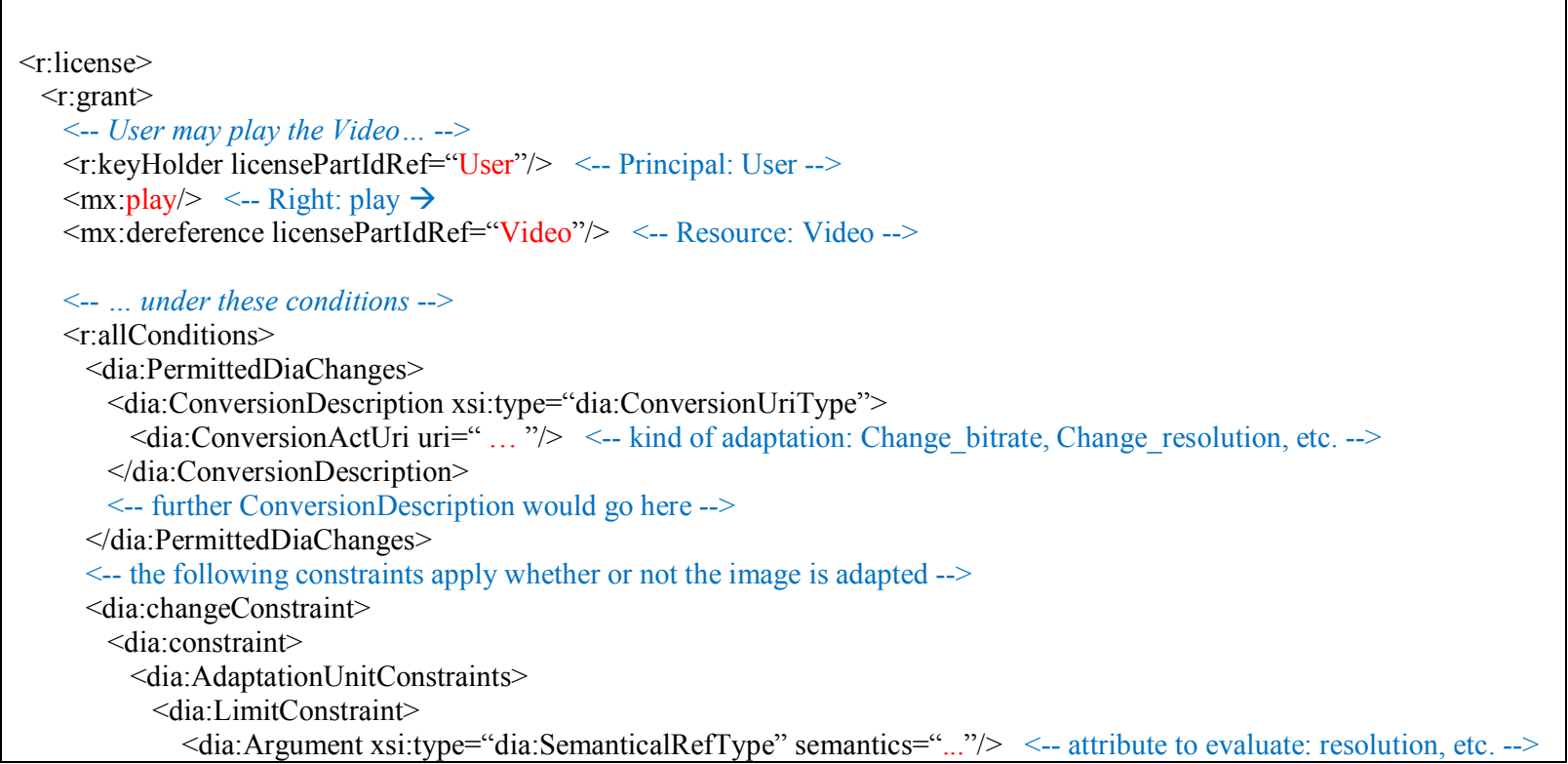




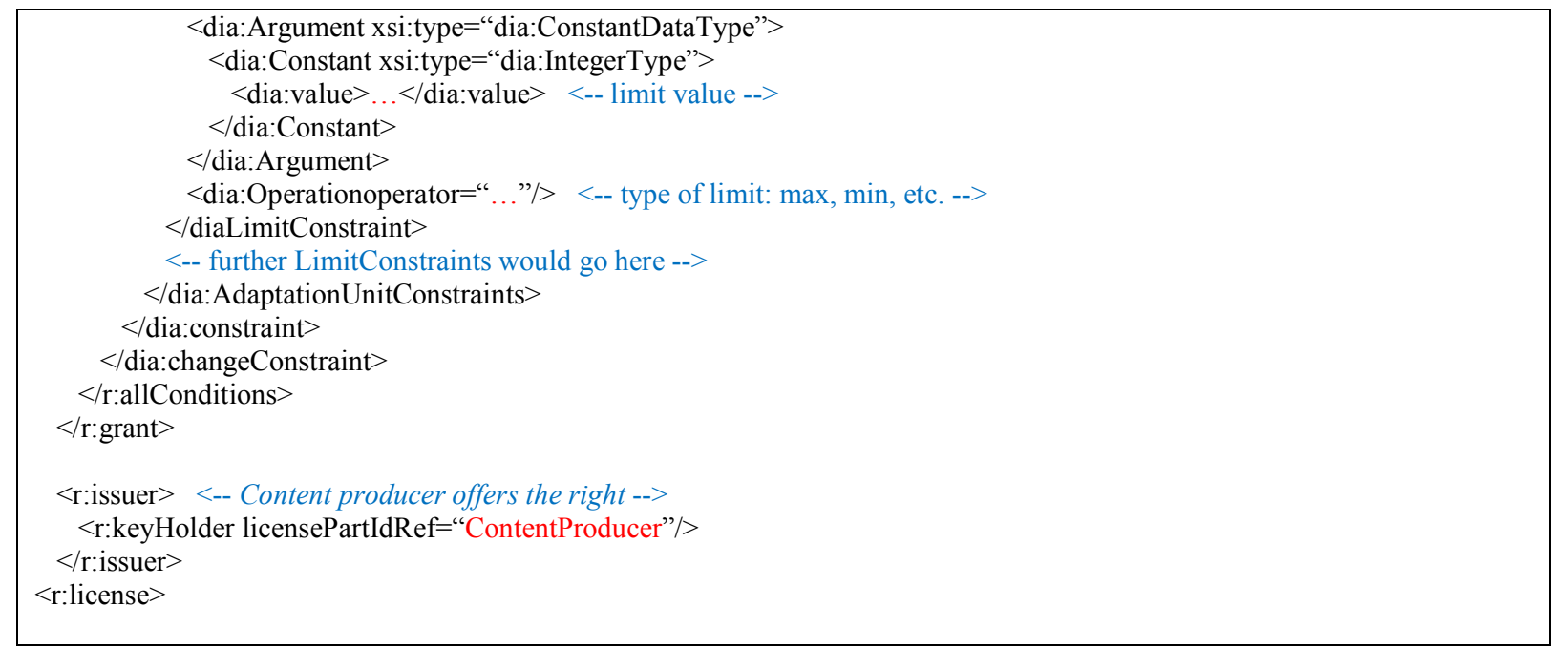

In addition and aligned to the context profiles presented in the previous subsection, we have defined a new profile to allow using the authorization information as a new type of context. Fig. 1 depicts the visualization of the XML schema of this new profile, named AuthorizationProfile, which contains two main elements: PermittedDiaChanges and ChangeConstraint. Its application will be presented in Section VI.

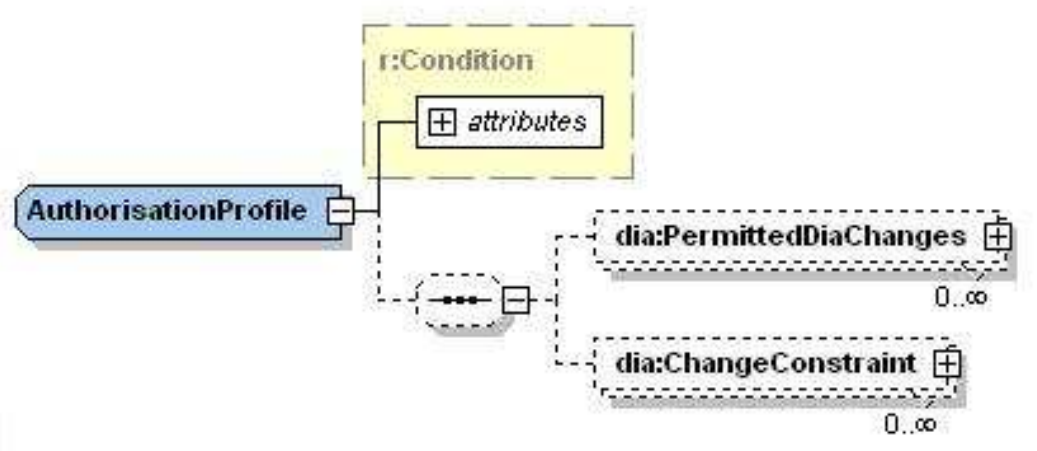

Fig. 1. Authorization profile

\section{USING ONTOLOGIES WITHIN THE ADAPTATION DECISION}

An ontology is used to define knowledge about a domain in terms of concepts, their characteristics and relationships or rules, providing formal and explicit descriptions of situations in that domain [33]. These descriptions are inferred by establishing logical relations among acquired context. They are similar to the descriptions that humans build, and yet can be used by 
machines. Decision taking operations that use an ontological framework to describe the context and relationships have thus higher chances to deliver decisions closer to the human way of reasoning, and hence to the users' expectations [5]. They are triggered by low-level context generated by sensors, notifying changes in the consumption environment. However, they take the decision using the additional knowledge that is inferred from the sensed data using the mechanisms provided by the ontology. Examples of low-level context have been indicated in Section II.A. Other examples are provided in Section VI.D.

In our work, we have developed a two-layer ontology adopting a methodology proposed in the literature, which is referred to as the Context-Aware Ontology for Multimedia Applications (MulTiCAO). Its model is shown in Fig. 2, and is developed using the Web Ontology Language (OWL) [34].

The core ontology layer (CAO.owl) provides the means to describe generic concepts and relationships that can be used and shared in any multimedia consumption application. Its design follows the profiling work described in Section III.A. In turn, these profiles are based on the classes defined in the UED tool provided by MPEG-21 DIA as well as on MPEG-7 MDS. Accordingly, the core ontology defines five basic concepts: User, Terminal, Network, Natural Environment and Media. More details concerning the conceptualization of the UED classes are provided in [35]. These concepts are found in any networked multimedia application. The advantage of using an ontology to represent them is the possibility of knowledge sharing between different domains, thus promoting interoperability and the possibility of inferring new knowledge.

On the other hand, the extended ontology is domain or application specific. It is developed as a collection of different domain-specific ontologies. Depending on the application, the combination of one or more of these specific ontologies may be used. In Fig. 2, two such specific-domain ontologies are represented. The CAO-aa describes adaptation authorization concepts and relations, whereas the CAO-rules provides the means to define and select possible adaptation operations that are most adequate to the application in view. In our work, targeting collaborative applications, both ontologies contain rules and relations suitable to this kind of applications, and hence they are both used. The extended context ontology allows building a context-aware system capable of formulating an authorized adaptation decision for collaborative applications, based on the changes of the contextual information data defined in the core context knowledge. This provides the means to simulate a governed network, where adaptation operations are only performed if they do not violate any condition expressed in DRM licenses. 


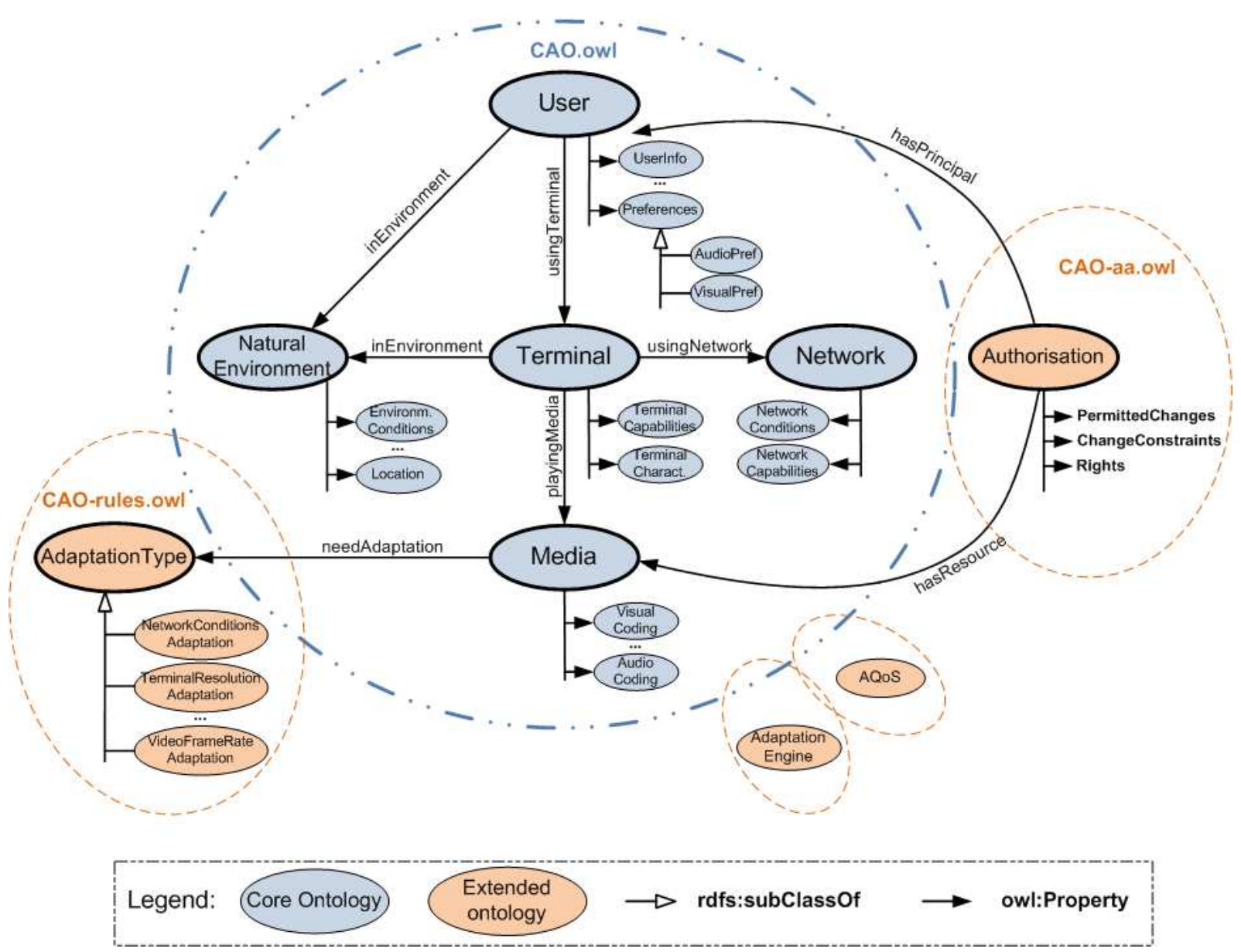

Fig. 2. MulTiCAO ontology model

The formulation of application specific rules in the extended ontology, enables to infer from the core knowledge (the sensed context), real-world situations that are indeed likely to occur in those specific applications, and accordingly to formulate the need for a specific adaptation. This inference process is performed by a rule-based engine module. New facts inferred by the rulebased engine enable the domain application to take an adaptation decision. Due to the considerable number of variables in a multimedia content consumption scenario, it is possible that multiple adaptation decisions may be plausible candidates or even that an ambiguous decision can be taken. It is thus necessary that the domain application provides a priority decision algorithm that best suits the user experience. This prioritization ensures that, among the possible adaptations that satisfy the constraints of the context situation, the system selects the one that maximizes the user satisfaction. 


\section{ARCHITECTURE OF THE VISNET II NOE CONTEXT-AWARE CONTENT ADAPTATION PLATFORM}

The context-aware content adaptation platform considered and developed within VISNET II NoE for a Virtual Classroom application, in which students are enabled to join in various classroom or lecture sessions remotely, is conceptually illustrated in Fig. 3. This platform is designed with view to context-aware adaptation of the delivered learning material. It also encompasses the use of rights management on the content to allow controlled dissemination to a heterogeneous audience. In combination, these features are envisaged to enable academic institutions to conduct a series of collaborative lectures and classes with which remotely located students can interact more efficiently.

In this setup, when one institute conducts a lecture in one of their lecture theatres for the enrolled students, those from other universities who have also enrolled to the same course can attend the same lecture from other campuses remotely. Unlike a conventional classroom session, these lecture series can also be followed by external students, those who have been unable to attend the classroom as well as the general public over wired or wireless links using their home desktop computers or mobile terminals, such as smart phones. Enrolled remote students can interact with the lecturer and audiences using the Virtual Collaboration facilities. In such a case, the delivered content is adapted to match individuals' preferences while also considering other related constraints. However, the general public has rights to view only a low resolution version of the learning material and do not have the privileges to view any of the adapted versions. Neither can they interact with the classroom sessions.

The proposed adaptation platform consists of the following four major modules: Adaptation Decision Engine (ADE), Adaptation Authorizer (AA), Context Providers (CxPs) and Adaptation Engine Stacks (AESs), which comprise a suite of Adaptation Engines (AEs).

These modules are independent units that interact with each other through Web Services-based interfaces. The distributed modular architecture of the adaptation platform ensures scalability. Well-defined interfaces based on open standards also guarantee interoperability and flexibility of freely adding, removing and migrating modules. The use of ontologies in the ADE, while being also a vehicle for interoperability, provides the platform with context-aware analysis capabilities closer to real-world situations. The AA ensures the governed use of protected contents. Flexible AESs enable the execution of a variety of adaptations that can be dynamically configured and requested on the fly. Thus, although it appears in Fig. 3 that this platform has a specific use focused on a Virtual Classroom scenario, it is indeed designed generic enough to serve for much bigger umbrella applications, such as a generic Virtual Collaboration application. The next subsections briefly describe the functionality of each module of this platform. 


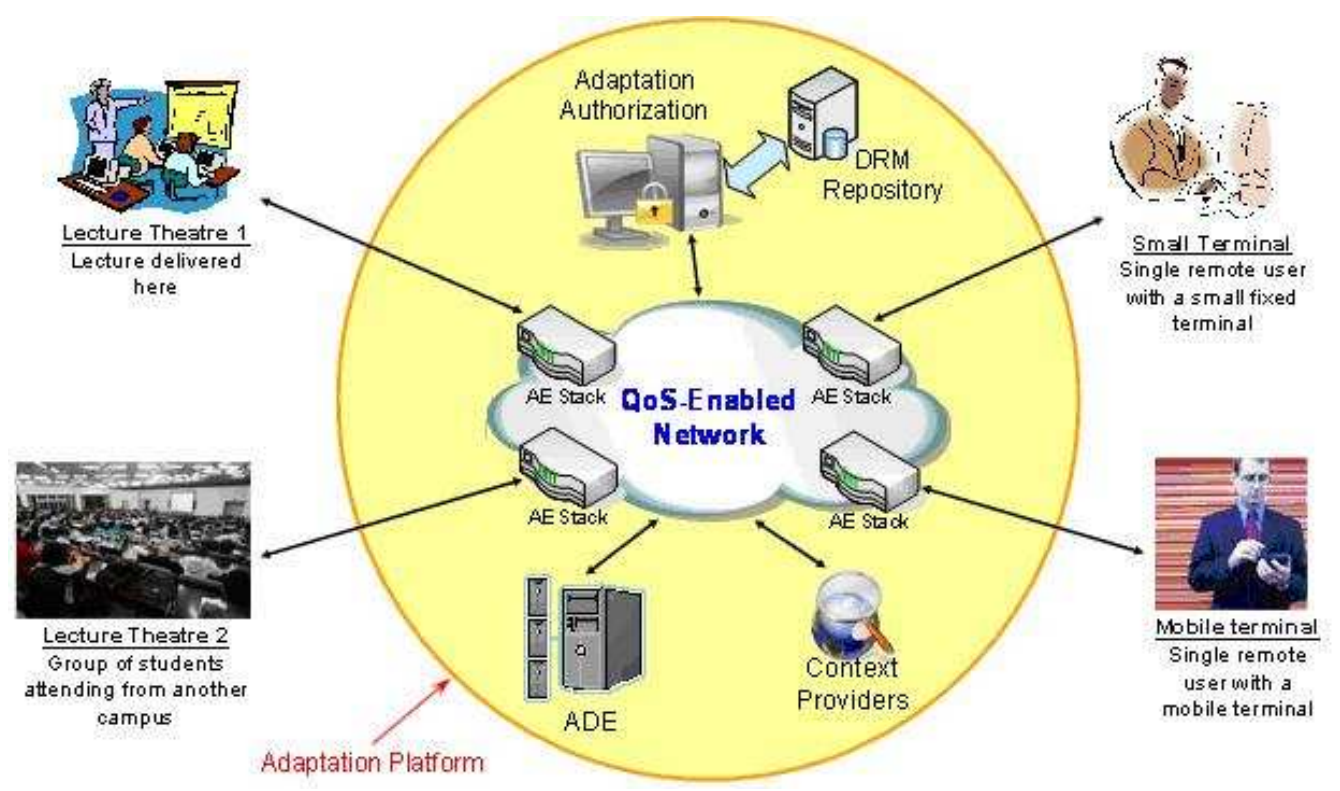

Fig. 3. VISNET II NoE context-aware content adaptation platform for a Virtual Classroom application

\section{A. Context Providers}

Entities, either software or hardware, that are able to generate and provide explicit contextual information are designated as Context Providers (CxPs). The low-level contextual information generated by these entities, once acquired and represented according to a standard format, is used to infer higher-level concepts, and thus assist the adaptation decision operation. Our platform uses the profiles based on MPEG-21 DIA UED specification to represent this context. With these profiles, each $\mathrm{CxP}$ needs only to know and implement its own sphere of action resulting in an enhanced level of interoperability.

The CxPs can be various in a complex application scenario. A few of the examples are:

- Network operators (through the network equipment).

- Content providers (through databases, media repositories, streaming servers, encoders, etc).

- Equipment manufacturers (through terminal devices, sensors, such as cameras, microphones, etc).

- Users (via the terminal device or databases holding user profiles).

The proposed platform exposes an Application Programming Interface (API) based on MPEG21 distinguishing the different profiles as identified in this paper, which are expected to be used by these CxPs accordingly. 


\section{B. Adaptation Decision Engine}

The Adaptation Decision Engine (ADE) is responsible for deciding how the system should react when changes in the context of usage are likely to affect the user's quality of experience. The architecture of the ADE middleware being designed in VISNET II NoE is illustrated in Fig. 4. It is based on a multi-module service-oriented approach, where each module provides a specific functionality or service. It is divided into three basic blocks: a) acquisition and initial processing of low-level context; b) ontology instantiation and merging; and c) reasoning and decision taking. The Context Service Manager gathers low-level context generated by external Context Providers (CxPs). It processes the incoming contextual messages, extracting relevant values to create an object model, which is stored in the Context Knowledge Base (KB). The Ontology Service Manager then instantiates this object model into the core ontology and integrates it with the concepts and inference rules defined in the Extended Ontology. It creates a structure of interrelated concepts, which is used by the Adaptation Decision Manager to infer situations, and accordingly to take the adaptation decision. This block also checks the consistency of the instantiated ontology. If erroneous information is presented or if information is missing, it sends back an error message. Only permitted adaptation operations and associated change constraints are instantiated in the ontology, restricting the adaptation decision possibilities. Whenever new or updated contextual information is available, the Adaptation Decision Manager invokes the Rules Inference Engine which interacts with the Decision Taking Engine module to select the most appropriate adaptation and corresponding service parameters.

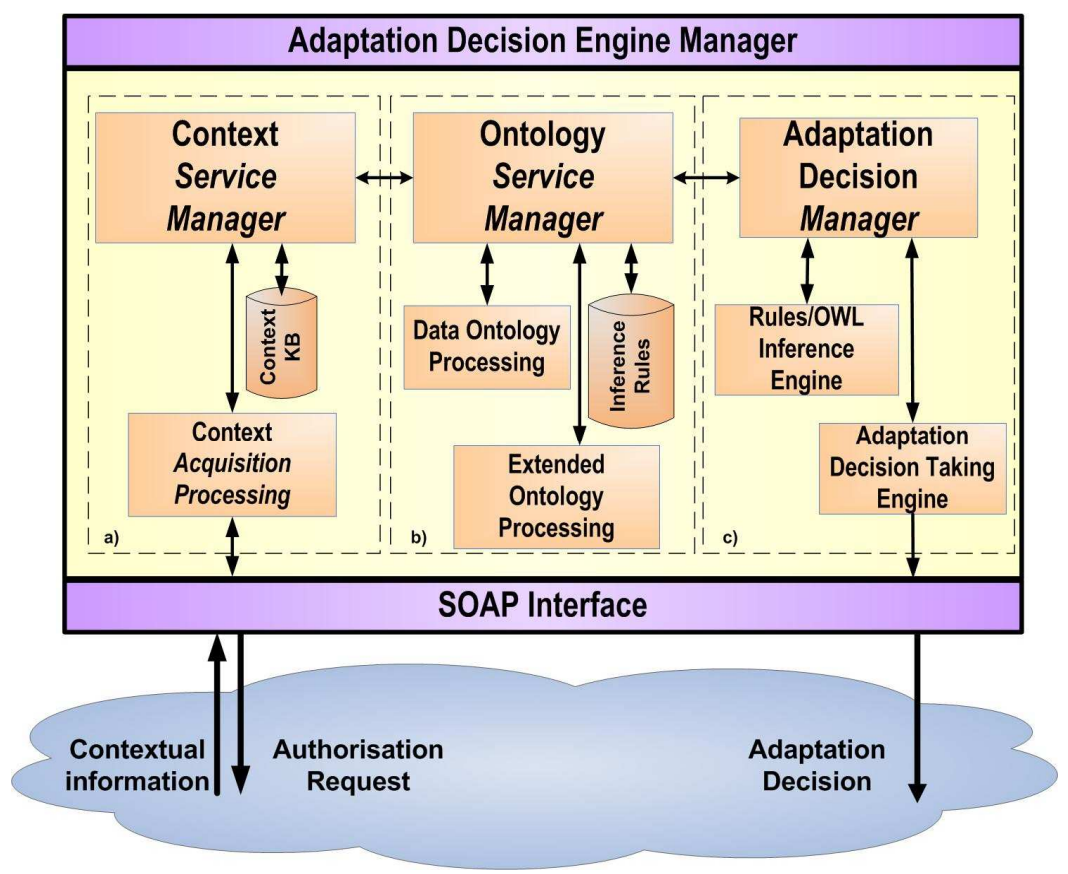

Fig. 4. Adaptation Decision Engine 
The ontology being used in this architecture was presented in Section IV. The inference rules, defined in the extended ontology concerning adaptation operations, must also reflect a prioritization scheme whereby clear decisions can be taken even in the presence of multiple possible adaptations satisfying the low-level context constraints. One of the greatest challenges in this architecture resides in the Adaptation Decision Taking module, whose functionality lies within the rules deployment.

\section{Adaptation Authorizer}

The main role of an Adaptation Authorizer (AA) in a governed system is to allow (or disallow) adaptation operations based on whether they violate any conditions expressed in the licenses. The AA acts as a "Context Provider (CxP)", converting licenses into adaptation constraints. This new type of contextual information is formatted and represented using the AuthorizationProfile (Fig. 1). The AA looks into the DRM repository (Fig. 3) to find all the licenses associated with a certain resource and user, and passes relevant adaptation constraints to the Adaptation Decision Engine (ADE), so that it can take an appropriate adaptation decision.

The adaptation authorization concept improves the adaptation efficiency by excluding some of the available adaptation options during the decision taking process under certain conditions. Although limiting some conversion options with the use of an AA may prevent the adaptation platform from responding to certain usage environment constraints effectively, such restrictions may be necessary to protect the legitimate rights of the content owners or producers.

\section{Adaptation Engine Stacks}

The Adaptation Engine Stacks (AESs) considered in this platform are capable of performing multiple adaptations. An AES encloses a number of Adaptation Engines (AEs) [36], [37] into a single entity, as illustrated in Fig. 5. All of the AEs in an AES reside in a single hardware platform sharing all the computational resources. The advantage of such an approach is that it is possible to cascade multiple AEs optimally to minimize the associated computational complexity. For example, if both cropping and scaling operations are needed to be performed on a given non-scalable video stream, those operations can be performed together in a cascaded fashion to improve the adaptation efficiency. In such a scenario, if both adaptations are performed together in a single module in the form of cascaded adaptation, much of the common tasks can be shared between the two adaptation operations. Parsing a bit stream to extract the necessary syntax elements for performing the adaptation operations and reassembling the adapted bit stream are two such tasks that can be shared. Moreover, deploying multiple AEs in a cascaded way, as opposed to using them located at various network nodes, helps to minimize the communication delay by avoiding the forwarding of media content from one AE to another. In this way, the potential negative effects of the extra delay incurred during content forwarding are avoided in a conversational application, such as Virtual Collaboration, which in turn leads to improved adaptation efficiency while enhancing quality of experience.

The AES has been organized in a three-layer architecture. The lowest layer is the Simple Object Access Protocol (SOAP) Interface, which is responsible for communication 
functionalities with the other interacting entities. The Processing and Selection Layer handles the system management functions. The Service Initialization Agent is responsible for initializing each component in the AES. The Registering Agent communicates with the Adaptation Decision Engine (ADE) to register its services, capabilities and required parameters. It is also responsible for renewing the registered information in case of any change in its service parameters. The Adaptation Decision Interpreter processes the adaptation decision message from the ADE requesting the adaptation service. Based on this information, it also decides on the appropriate $\mathrm{AE}$ to be invoked and its configurations. The progress of the adaptation operation is monitored by the AE Monitoring Service and if necessary, it informs the ADE about the progress.

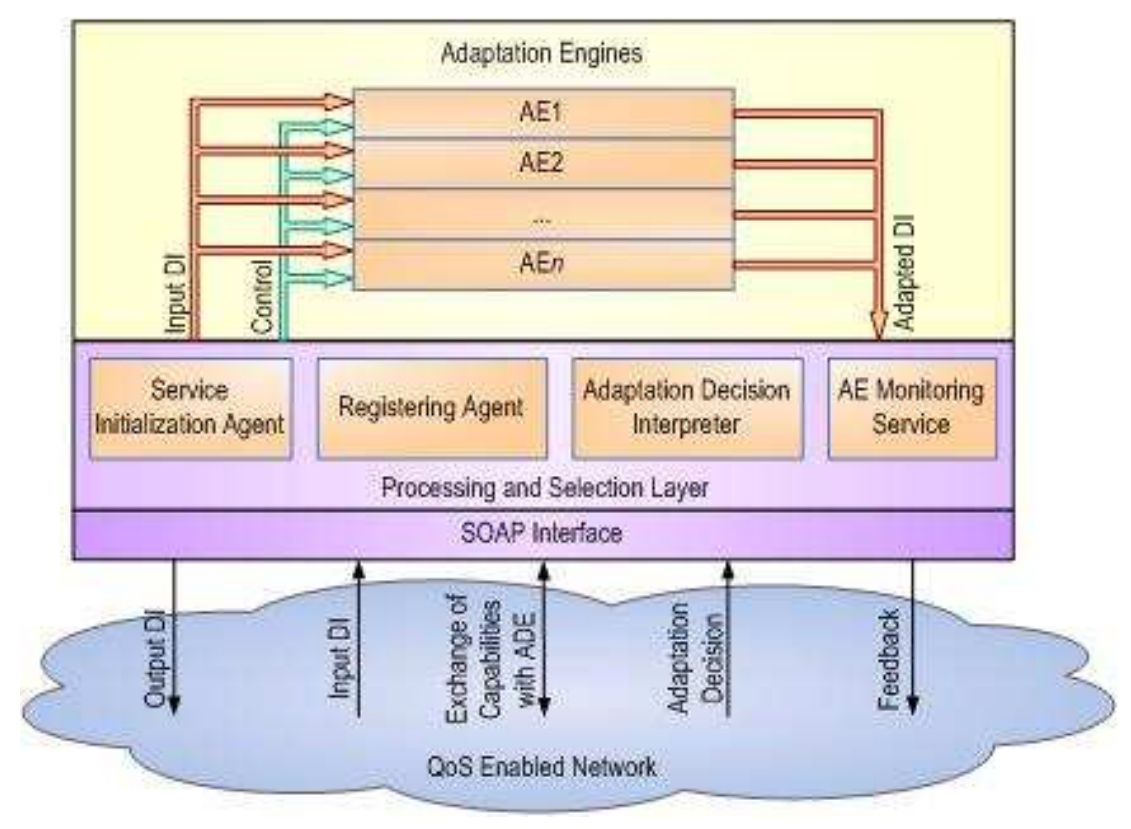

Fig. 5. Adaptation Engine Stack

\section{INTERFACES BETWEEN MODULES IN THE VISNET II NOE ADAPTATION PLATFORM}

This section provides the insights into the interfaces required between the modules as part of the proposed content adaptation platform and the sequence of events that take place while performing DRM-based adaptation for a particular user or group of users. Fig. 6 presents the functional architecture of this platform, in which the interfaces between modules are illustrated. For this distributed environment, the exchange of messages is addressed using SOAP, a simple and extensible Web Services protocol. 


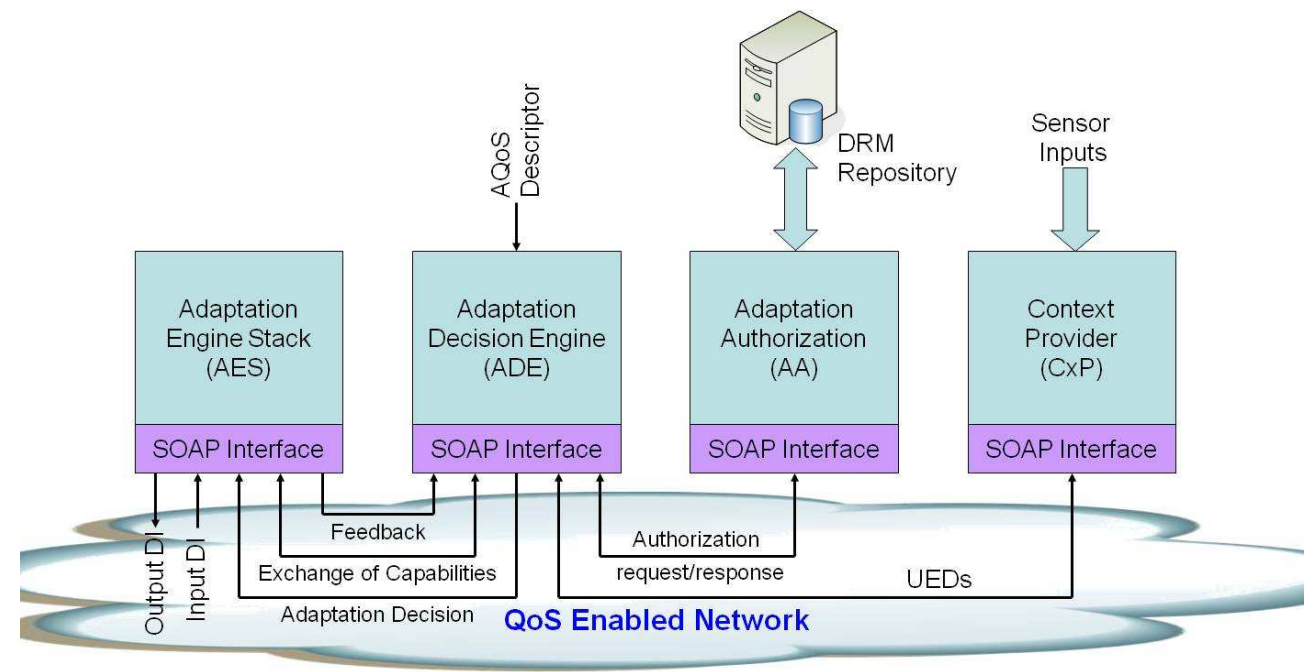

Fig. 6. Functional architecture of the platform

The operation of the context-aware content adaptation platform is divided into four stages, as depicted in Fig. 7. The first stage is known as the System Initialization Phase and during this phase, system initialization functions are carried out. During the Service Negotiation Phase, service parameters are determined for a given user. During the Service Initialization Phase, the system is configured to offer the service. The stage when the service is offered is known as the In-Service Phase. These phases may co-exist at a given instance of time when multiple users are considered. 


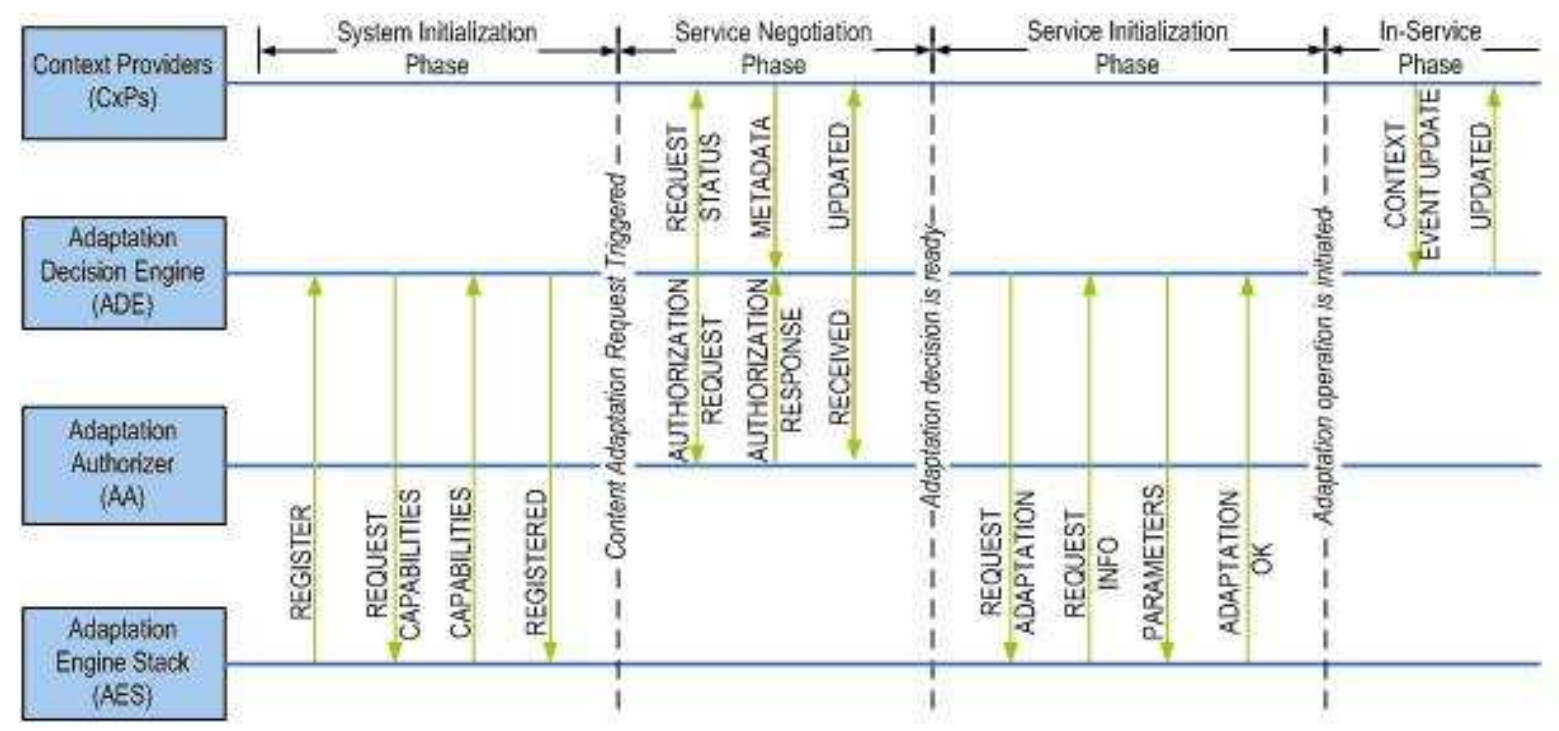

Fig. 7. Parameter exchange

\section{A. Adaptation Decision Engine-Context Providers interface}

To obtain low-level context information, the Adaptation Decision Engine (ADE) can either query Context Providers (CxPs) or listen for events sent by CxPs depending on the service status. During the service negotiation phase, the ADE queries the CxPs, as shown in Fig. 7. The received contextual information is extracted from the context profiles defined and registered in the ontology model. After the service is launched, the CxPs work in a "push" mode notifying the ADE when new context is available via the context update message illustrated in Fig. 7. In this way, the ADE is enabled to react to any significant changes in context, adjusting the adaptation parameters accordingly.

\section{B. Adaptation Decision Engine-Adaptation Engine Stack interface}

While designing the interface between the Adaptation Engine Stack (AES) and Adapation Decision Engine (ADE), factors such as ability to have multiple AESs operating within the system and their ability to join, leave and migrate seamlessly are considered. This flexibility is ensured by the implementation of a dedicated system initialization phase, whereby a sequence of messages is exchanged between the ADE and AES, as illustrated in Fig. 7. The parameters exchanged inform the ADE of the AES's adaptation capabilities and necessary metadata related to those capabilities, e.g., maximum and minimum bit rates, maximum spatial resolution, etc, along with the registration request. In order to conclude the registration on the ADE database, the AES should also inform the ADE of its IP address and the service identifier. Once the registration phase is completed, the AES is ready to perform adaptation operations when the $\mathrm{ADE}$ requests. An example of the registration request message is shown in Table II.

During the service initialization phase, in line with the decision taken, the ADE requests the 
AESs to invoke the selected adaptation operations on the original DI and forward the adapted DI to the user, as shown in Fig. 7. This request also contains the related adaptation parameters including the source DI, desired adaptation operations and associated metadata.

\section{Adaptation Decision Engine-Adaptation Authorizer interface}

When dealing with protected content, the Adaptation Decision Engine (ADE)'s content adaptation decision is preceded by an authorization request, which identifies the User that consumes the adapted content and the multimedia resource, which is going to be adapted, by its DI Identifier. Sequence of messages exchanged between the Adaptation Authorizer (AA) and ADE to obtain permitted adaptation operations is illustrated in Fig. 7. Once the AA receives the authorization request from the $\mathrm{ADE}$, it responds with all the adaptation related information contained in the license associated to the referred multimedia resource and User. This information includes the permitted adaptation operations as well as the adaptation constraints associated to those operations. Both the permitted adaptation operations and related constraints are expressed in a format compatible to MPEG-21 DIA.

\section{Parameter exchange based on a selected application scenario}

This subsection details the sequence of messages exchanged between the modules of the content adaptation platform based on an example use case described below.

\section{Virtual Classroom application scenario}

In the selected use case, a student wishes to attend a Virtual Classroom session of one hour, using his/her Personal Digital Assistant (PDA) over a $3^{\text {rd }}$ Generation (3G) mobile network. The requirements imposed by the application to offer the best quality of the Virtual Classroom multimedia material indicate a bit rate of $1 \mathrm{Mbps}$ and a spatial resolution of $640 \times 480$ pixels. During this use case, the student moves from the faculty campus to outside. He/she walks down the street and enters a railway station, crowded with people. The student sits there for a few minutes to wait for a friend to arrive on the 5 o'clock train. In this noisy environment, not only will the network connectivity be worse due to mobile network conditions, but also it will prove harder to hear, watch and follow the classroom session, as the student will be surrounded by many people at the station. We assume that the Virtual Classroom session in this particular use case includes a lab demonstration describing a human anatomical body model. Considering the reduced display dimensions of the PDA, the Adaptation Decision Engine (ADE) could decide to perform automatic cropping of the head-and-shoulders to enhance the visibility of the lab demonstrator in action, particularly in such a noisy environment. However, such adaptation would then exclude the body model under demonstration from the view of the student, which would be equally important for the completeness of the information. Therefore, not authorizing such an adaptation, which would otherwise impair the comprehension of the content, but enforcing the ADE to take a different decision (e.g., changing the focus of attention) will lead to an improvement in the overall quality of experience. Additionally, the extended ontology also plays an important role here in assisting the selection of the best adaptation among a set of adaptations that satisfy the usage constraints. This is illustrated in the following paragraphs. 


\section{Parameter exchange}

The sequence of messages transferred between the modules for performing content adaptation in the aforementioned use case is summarized in the sequence chart shown in Fig. 7.

Table II. Contents of the AES registration request message

\begin{tabular}{|l|l|}
\hline \multicolumn{1}{|c|}{ Parameters } & \multicolumn{1}{c|}{ Metadata } \\
\hline Time & Tue, 29 Jan 2008 15:32:58 \\
\hline Multimedia content identifier & MPEG-21 DI \\
\hline IP Address & 202.145.2.98 \\
\hline Service identifier & Service ID of the AES \\
\hline \multicolumn{2}{|c|}{ Capabilities } \\
\hline Spatial resolution scaling & Scalable \\
\hline Temporal resolution scaling & Scalable \\
\hline Bit rate scaling & Scalable \\
\hline ROI Cropping & $\begin{array}{l}\text { Maximum spatial resolution }=720 \times 560 \text { pixels } \\
\text { Minimum spatial resolution }=16 \times 16 \text { pixels } \\
\\
\text { Maximum temporal resolution }=50 \text { fps } \\
\text { Minimum temporal resolution }=0 \text { fps } \\
\text { Maximum cropping window size }=720 \times 560 \text { pixels } \\
\text { Minimum cropping window }=16 \times 16 \text { pixels }\end{array}$ \\
\hline & $\begin{array}{l}\text { Transmoding languages: English, Spanish, } \\
\text { Portuguese } \\
\text { Display font sizes: small, medium, large } \\
\text { Display position: adaptive, coordinates on the } \\
\text { display }\end{array}$ \\
\hline
\end{tabular}

The Virtual Classroom administrator notifies the ADE when a student joins the Virtual Classroom session. During this service negotiation phase, the ADE queries the Context Providers (CxPs) for context information associated with the new user. It then requests the Adaptation Authorizer (AA) to provide information on the permitted adaptation operations upon the available Virtual Classroom materials for the particular user. Contents of contextual messages received by the ADE are listed in Table III. Table IV shows the MPEG-21 DIA formatted message with the authorization information. 
Table III. Context information received

\begin{tabular}{|c|c|c|}
\hline & Parameters & Metadata \\
\hline \multirow{6}{*}{$\begin{array}{l}\text { Context information } \\
\text { received from the } \\
\text { terminal (From CxP } \\
\text { to } \mathrm{ADE} \text { ) }\end{array}$} & Time & Tue, 29 Jan 2008 15:32:58 \\
\hline & Context provider identifier & Terminal ID \\
\hline & Terminal capabilities & $\begin{array}{l}\text { Display size }(\text { height } \times \text { width })=\text { QCIF } \\
(176 \times 144 \text { pixels })\end{array}$ \\
\hline & Terminal capabilities & Maximum frame rate $=25 \mathrm{fps}$ \\
\hline & Terminal capabilities & BatteryTimeRemaining $=35$ minutes \\
\hline & Terminal capabilities & $\begin{array}{l}\text { Codecs supported = MPEG-4, } \\
\text { H.264/AVC }\end{array}$ \\
\hline \multirow{3}{*}{$\begin{array}{l}\text { Context information } \\
\text { received from the } \\
\text { network service } \\
\text { provider (From CxP } \\
\text { to ADE) }\end{array}$} & Time & Tue, 29 Jan 2008 15:32:58 \\
\hline & Context provider identifier & Network service provider's ID \\
\hline & Network conditions & Available bandwidth $=228 \mathrm{kbps}$ \\
\hline \multirow{7}{*}{$\begin{array}{l}\text { Adaptation } \\
\text { authorization } \\
\text { response (From AA } \\
\text { to ADE) }\end{array}$} & Time & Tue, 29 Jan 2008 15:32:58 \\
\hline & Multimedia content identifier & MPEG-21 DI \\
\hline & Type of user identifier & MPEG-21 KeyHolder \\
\hline & \multicolumn{2}{|c|}{ Possible Adaptation Operations } \\
\hline & Spatial resolution scaling & $\begin{array}{l}\text { Minimum SpatialResolution }=150 \times 100 \\
\text { pixels }\end{array}$ \\
\hline & Temporal resolution scaling & Minimum TemporalResolution $=10 \mathrm{fps}$ \\
\hline & Bit rate scaling & Minimum nominal bit rate $=30 \mathrm{kbps}$ \\
\hline
\end{tabular}

Table IV. An extract from an MPEG-21 DIA formatted authorization response

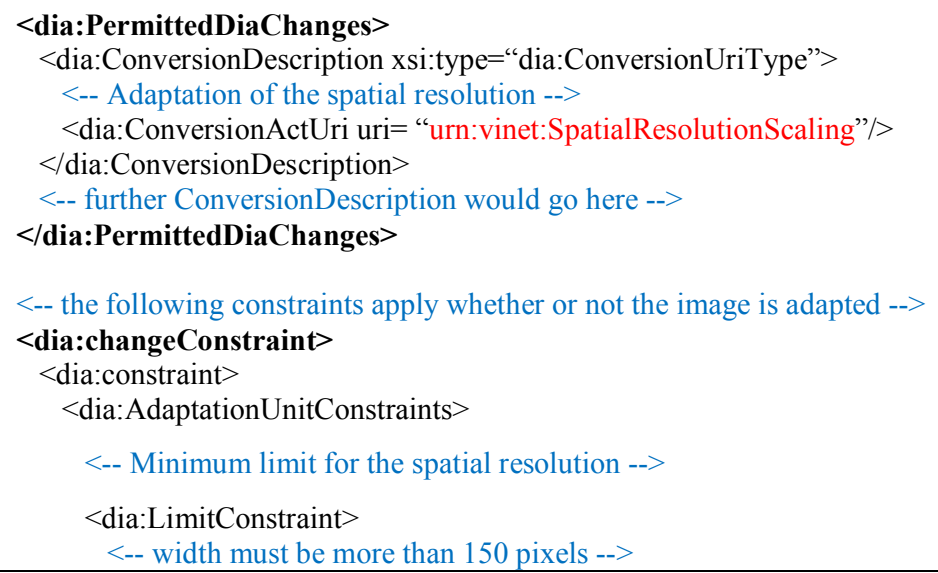




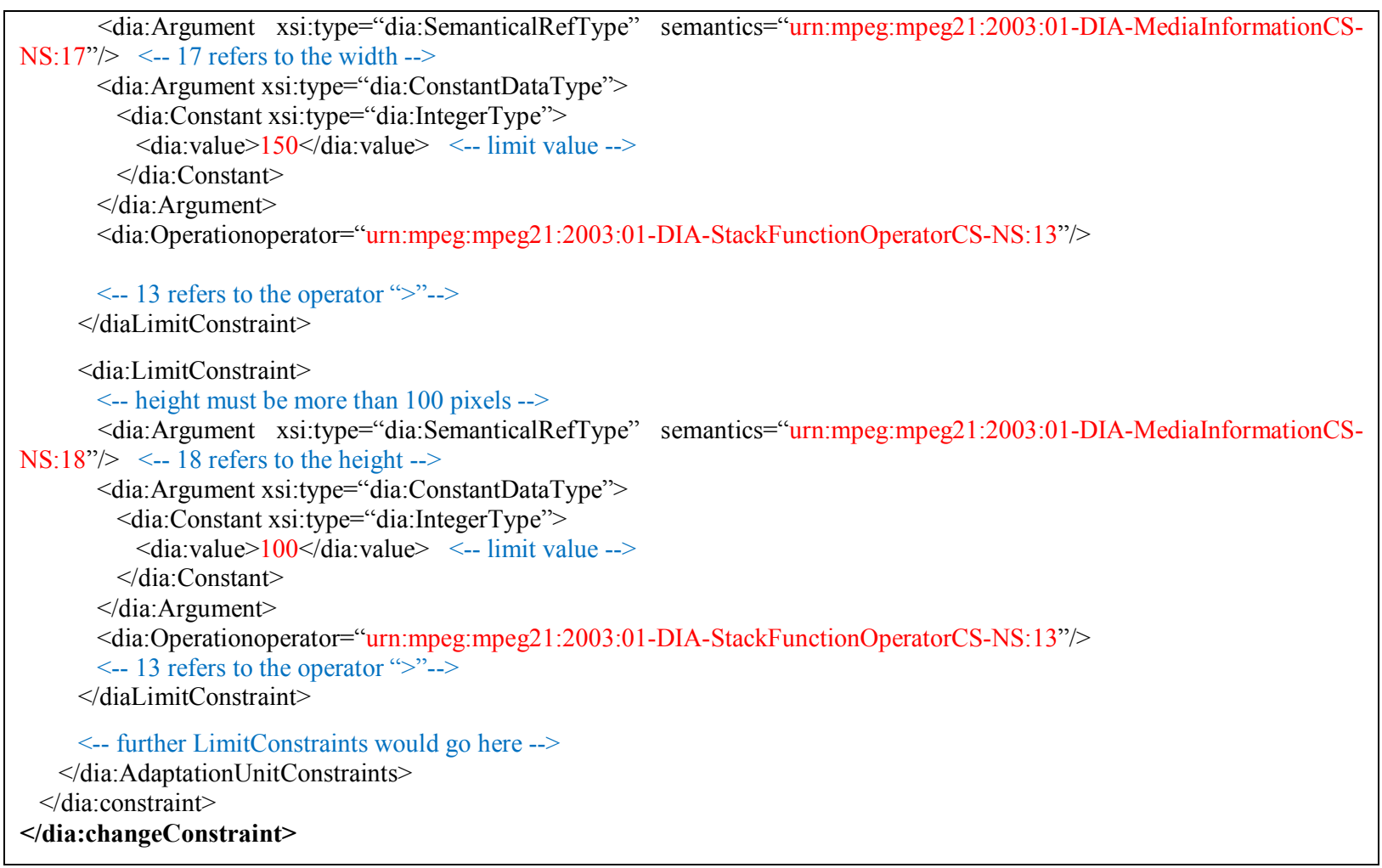

The terminal context reveals that the device has a small display and that its remaining battery lifetime is limited. As a result, the ADE decides to downscale the video resolution by a factor of four from the original resolution down to $160 \times 120$ pixels. This adaptation fits into the Quarter Common Intermediate Format (QCIF) display size of the PDA adequately, while also staying within the authorized resolution limits specified by the AA (i.e., $150 \times 100$ pixels). To satisfy the restrictions concerning the battery power, the ADE decides to request a summarization of the video. The network context indicates that available resources are not sufficient to support the best video quality. To respond to this constraint, the ADE decides to downscale the temporal resolution of the video. Another possible adaptation to perform could be to simply decrease the bit rate, which would have an impact on the picture quality. However, given the kind of application being addressed - the Virtual Classroom scenario - the ADE is capable to infer that decreasing the temporal resolution will provide a better quality of experience to the user than to deteriorate the overall picture quality. Picking up on the above referred example, namely a lab demonstration describing a human anatomical body model, it is clear for humans that higher picture fidelity is more important to the overall comprehension of the content than a full temporal resolution video. This kind of extra knowledge is provided to the application by the specific ontology in the extended layer.

Once the ADE takes the adaptation decision, it contacts the appropriate Adaptation Engine Stacks (AESs), passing the selected adaptation parameters, as shown in Table V. This concludes 
the service negotiation phase, placing the system in a position to serve the user's request in the best possible way, while satisfying his/her initial context constraints. During the in-service phase, the ADE keeps on monitoring the dynamics of the context through the context update information received from the CxPs. Significant changes affecting the user's satisfaction, such as increased bandwidth restrictions or high noise level, trigger a new adaptation decision process. If there is any better set of adaptation operations, then the ADE reconfigures the involving AESs accordingly through another content adaptation request. To cope with the referred context changes during the in-service phase, possible adaptations would include the conversion of audio to text (or eliminating the audio component) and the further decrease of the video temporal resolution.

Table V. Adaptation parameters

\begin{tabular}{|l|l|}
\hline \multicolumn{1}{|c|}{ Parameters } & \multicolumn{1}{c|}{ Metadata } \\
\hline Time & Tue, 29 Jan 2008 15:32:58 \\
\hline Multimedia content identifier & MPEG-21 DI \\
\hline Display size & QCIF (176×144 pixels) \\
\hline \multicolumn{2}{|c|}{ Required Adaptations Operations } \\
\hline Spatial resolution scaling & $\begin{array}{l}\text { Spatial resolution before adaptation }=\text { VGA }(640 \times 480) \\
\text { Spatial resolution after adaptation }=\text { Quarter QVGA } \\
(160 \times 120)\end{array}$ \\
\hline Temporal resolution scaling & $\begin{array}{l}\text { Temporal resolution before adaptation }=25 \mathrm{fps} \\
\text { Temporal resolution after adaptation }=12.5 \mathrm{fps}\end{array}$ \\
\hline Bit rate scaling & $\begin{array}{l}\text { Bit rate before adaptation }=1 \mathrm{Mbps} \\
\text { Bit rate after adaptation }=128 \mathrm{kbps}\end{array}$ \\
\hline
\end{tabular}

\section{CONCLUSION}

This paper has presented the concepts and architecture of a proposed scalable and modular platform for context-aware and DRM-enabled content adaptation. The proposed platform enables users to access and exchange pervasive yet protected and trusted content seamlessly using diverse terminal and connectivity types while taking their preferences, geographical locations and environments also into account. The applicability of this platform has been presented in the context of a Virtual Classroom application scenario, which is assumed to be a particular education-orientated use case for a more generic collaboration environment. It has been discussed that by integrating context and context-related issues together with adaptation, it is possible to better serve various dynamic requirements arising from diverse usage environments. While doing this, it has been demonstrated that the combined use of ontologies and low-level contextual information can facilitate the adaptation decision process, which is the heart of the adaptation platform. Verifying and enforcing usage rights within the adaptation operations have also been highlighted, and a novel context profile has been created for the purpose of authorizing 
the adaptation. It is envisaged that the integrated operation of the several modules that compose such a modular architecture with well-defined and standards-based interfaces will greatly contribute to the interoperability and scalability of future media content delivery systems. Current state-of-the-art research on context-awareness systems is also investigating such aspects, albeit typically these tend to be dealt with in an isolated manner, as opposed to the comprehensive platform proposed in this paper.

\section{ACKNOWLEDGMENT}

The work presented was developed within VISNET II, a European Network of Excellence (http://www.visnet-noe.org), funded under the European Commission IST FP6 programme.

\section{REFERENCES}

[1] Y. Wang, J.-G. Kim, S.-F. Chang, and H.-M. Kim, "Utility-based video adaptation for Universal Multimedia Access (UMA) and content-based utility function prediction for realtime video transcoding," IEEE Trans. Multimedia, vol. 9, no. 2, pp. 213-220, Feb. 2007.

[2] A. Vetro, "MPEG-21 digital item adaptation: Enabling universal multimedia access," IEEE Multimedia J., vol. 11, no. 1, pp. 84-87, Jan.-Mar. 2004.

[3] A. Carreras and J. Delgado, "A new type of contextual information based on the adaptation authorisation," in Proc. $9^{\text {th }}$ Int. Workshop on Image Analysis for Multimedia Interactive Services (WIAMIS 2008), Klagenfurt, Austria, 7-9 May 2008.

[4] "VISNET II NoE," 2007. [Online]. Available: http://www.visnet-noe.org/.

[5] M.T. Andrade, H. Kodikara Arachchi, S. Nasir, S. Dogan, H. Uzuner, A.M. Kondoz, J. Delgado, E. Rodriguez, A. Carreras, T. Masterton, and R. Craddock, "Using context to assist the adaptation of protected multimedia content in virtual collaboration applications," in Proc. $3^{\text {rd }}$ IEEE International Conference on Collaborative Computing: Networking, Applications and Worksharing (CollaborateCom'2007), New York, NY, USA, 12-15 Nov. 2007.

[6] B. Schilit, N. Adams, and R. Want, "Context-Aware Computing Applications," in Proc. IEEE Workshop on Mobile Computing Systems and Applications, Santa Cruz, USA, pp. 8590, December 1994.

[7] A.K. Dey, "Providing Architectural Support for Building Context-Aware Applications," Ph.D. Thesis, College of Computing, Georgia Institute of Technology, Atlanta, Georgia, 2000.

[8] N. Ryan, J. Pascoe, and D. Morse, "Enhanced reality fieldwork: the context-aware archaeological assistant", V. Gaffney, M. van Leusen, S. Exxon (eds), Computer Applications in Archaeology, 1997.

[9] R. Hull, Ph. Neaves, J. Bedford-Roberts, "Towards situated computing", in Proc. International Symposium on Wearable Computers, 1997.

[10]P.J. Brown, "The Stick-e Document: a framework for creating context-aware applications", in Electronic Publishing, Palo Alto, 1996. 
[11]J.L. Crowley, J. Coutaz, G. Rey, and P. Reignier, "PerceptualComponents for Context Aware Computing", in Proc. $4^{\text {th }}$ International Conference on Ubiquitous Computing , Vol.2498, pp.117-134, 2002.

[12] "Composite Capability/ Preference Profiles (CC/PP): Structure and vocabularies 1.0, Standard", W3C Recommendation, Jan. 2003.

[13]"UAProf, User Agent Profile", OMA Open Mobile Alliance (OMA) OMA-TS-UAProf-V2 0-20060206-A, 2006.

[14]World Wide Web Consortium [Online]. Available: http://www.w3.org/

[15]Open Mobile Alliance [Online]. Available: http://www.openmobilealliance.org/

[16]"Information Technology - Multimedia Framework (MPEG-21) - Part 7: Digital Item Adaptation," ISO/IEC Standard ISO/IEC 21000-7:2007, Dec. 2007.

[17]I. Burnett, F. Pereira, R. Walle, and R. Koenen eds., "The MPEG-21 Book," John Wiley and Sons Ltd, 2006.

[18]X. Wang, T. DeMartini, B. Wragg, M. Paramasivam, and C. Barlas, "The MPEG-21 rights expression language and rights data dictionary," IEEE Trans. Multimedia, vol. 7, no. 3, pp. 408-416, Jun. 2005.

[19]E. Rodríguez, "Standardisation of the protection and governance of multimedia content," Ph.D. Thesis, Department of Information and Communication Technologies, Universitat Pompeu Fabra, Barcelona, 2006.

[20]A. Vetro and C. Timmerer, "Digital item adaptation: Overview of standardization and research activities," IEEE Trans. Multimedia, vol. 7, no. 3, Jun. 2005.

[21]L. Rong and I. Burnett, "Dynamic multimedia adaptation and updating of media streams with MPEG-21," in Proc. $1^{\text {st }}$ IEEE Conf. Consumer Commun. Netw., pp. 436-441, Jan. 2004.

[22]J. Delgado, V. Torres, S. Llorente, and E. Rodríguez, "Rights and trust in multimedia information management," Lecture Notes in Computer Science (LNCS), Springer, vol. 3677, pp. 55-64, Sep. 2005.

[23]"Information Technology - Multimedia Framework (MPEG-21) - Part 5: Rights Expression Language," ISO/IEC Standard ISO/IEC 21000-5:2004, Mar. 2004.

[24]“DAIDALOS,” Dec. 2005. [Online]. Available: http://www.ist-daidalos.org

[25]"aceMedia," Dec. 2006. [Online]. Available: http://www.acemedia.org

[26]"AXMEDIS," 2007. [Online]. Available: http://www.axmedis.org

[27]"Projecte Integrat," Oct. 2005. [Online]. Available: http://www.i2cat.cat/i2cat/servlet/I2CAT.MainServlet?seccio=21_33.

[28]D. Rios, P. Dockhorn, G. Guizzardi, L. Ferreira, J. Pereira, and M. van Sinderen, "Using ontologies for modeling context-aware services platforms," in Proc. Workshop on Ontologies to Complement Software Architectures, Oct. 2004.

[29]T. Gu, H.K. Pung, and D.Q. Zhang, "A service-oriented middleware for building contextaware services," J. Netw. Comput. Appl., vol. 28, no. 1, pp. 1-18, Jan. 2005.

[30]W. Qin, Y. Shi and Y. Suo, "Ontology-based context-aware middleware for smart spaces," Tsinghua Science \& Technology, vol. 12, no. 6, pp. 707-713, Dec. 2007. 
[31]P. Dockhorn Costa, "Towards a services platform for context-aware applications," Masters Thesis, University of Twente, The Netherlands, 2003.

[32]"Information Technology - Multimedia content description interface - Part 5: Multimedia description schemes," ISO/IEC Standard ISO/IEC 15938-5:2003, 2003.

[33]A. Farquhar, R. Fikes, W. Pratt, and J. Rice, Collaborative ontology construction for information integration, Stanford University, Tech. Rep. KSL-95-63, Aug., 1995.

[34]"Web Ontology Language (OWL): Overview," W3C Recommendation, Feb. 2004. [Online]. Available: http://www.w3.org/TR/owl-features/.

[35] V. Barbosa, A. Carreras, H. Kodikara Arachchi, S. Dogan, M.T. Andrade, J. Delgado, and A.M. Kondoz, "A scalable platform for context-aware and DRM-enabled adaptation of multimedia content," in Proc. ICT Mobile and Wireless Communications Summit (ICTMobileSummit 2008), Stockholm, Sweden, 10-12 Jun. 2008.

[36]S. Eminsoy, S. Dogan, and A.M. Kondoz, "Transcoding based error-resilient video adaptation for 3G wireless networks," EURASIP J. Adv. Signal Process. (JASP), vol. 2007, article ID 39586, 13 pages, 2007.

[37]H. Kodikara Arachchi, S. Dogan, H. Uzuner, and A.M. Kondoz, "Utilising macroblock SKIP mode information to accelerate cropping of an H.264/AVC encoded video sequence for user centric content adaptation," in Proc. $3^{\text {rd }}$ Int. Conf. AXMEDIS 2007, Barcelona, Spain, Nov. 2007.

Biographies

Anna Carreras obtained her B.Sc. degree in Telecommunication Engineering in 2006 and her M.Sc. degree in Computer Architectures, Networks and Systems in 2008, both from Universitat Politècnica de Catalunya (UPC), Barcelona, Spain. Member of the Distributed Multimedia Applications Group (DMAG) since 2005. Her research interests include Digital Rights Management, and the use of context and metadata in distributed multimedia applications.

Vitor Barbosa is a research fellow at INESC Porto in the Telecommunications and Multimedia Unit. He obtained his M.Sc. in Electrical and Computers Engineering from the Faculty of Engineering, University of Porto (FEUP), Portugal, in 2008. His current research interests include multimedia systems and supporting technologies, multimedia metadata modelling, ontology engineering, Semantic Web, and context-aware content adaptation.

Hemantha Kodikara Arachchi received his B.Sc. (Eng.) degree with honors (1997), M.Phil. degree in Electronic and Telecommunication Engineering from University of Moratuwa, Sri Lanka (2000) and Ph.D. degree in Telecommunications from Asian Institute of Technology, Thailand (2004). Currently, he is a Research Fellow at I-Lab, University of Surrey, UK. His research interests are in video coding and context-aware content adaptation. 
Safak Dogan is a Senior Research Fellow at I-Lab, University of Surrey with M.Sc. and Ph.D. degrees in Satellite Communications (1996) and Multimedia Communications (2001), respectively. His research interests include video adaptation, transcoding, context-based content adaptation, low bit-rate video communications, error-resilience, and multi-party conferencing. $\mathrm{He}$ is a member of the IEEE and the EC Future Media and 3D Internet-Task Force.

Maria Teresa Andrade is a Professor at the Electrotechnical and Computing department of the Faculty of Engineering, University of Porto. She coordinates the Multimedia Systems group at INESC Porto. Her main interests are multimedia networking, content adaptation and contextawareness. Author of 40 published papers. Received her M.Sc. and Ph.D. degrees from the University of Porto.

Jaime Delgado received his Ph.D. degree in Telecommunication Engineering in 1987. Professor at the Computer Architecture Department of the UPC. Head and founder of the Distributed Multimedia Applications Group (DMAG).

Project Manager of European and national research projects. Editor of International Standards. Evaluator and reviewer for the European Commission and several Spanish Ministries. Author of hundreds of papers and books.

Eva Rodríguez obtained her B.Sc. degree in Telecommunication Engineer (2001) and her Ph.D. degree in Computer Science (2007). She is an Associate Professor at the Computer Architecture Department in the Universitat Politècnica de Catalunya (UPC). Her research interests include Digitals Rights Management and content protection. Active participation in MPEG-21 standard. Author of several published papers in international journals and conferences.

Ahmet M. Kondoz received his M.Sc. and Ph.D. degrees in Telematics (1984) and Communication (1986), respectively. He is a Professor in Multimedia Communication Systems at University of Surrey and the Head of I-Lab. He has graduated more than 40 Ph.D. students, and has been a consultant for major technology companies. He is a member of the IEEE and IET B.Eng. 